\title{
Inverse Scattering in Microwave Imaging for Detection of Malignant Tumor inside the Human Body
}

\author{
Muhammad Hassan Khalil and Xu Jiadong \\ Department of Electronics and Information Engineering \\ Northwestern Polytechnical University \\ Xi'an, China Postal Code, 710072 \\ hassan_janjua86@yahoo.com
}

\begin{abstract}
The detection of breast cancer in its early stage is a developing research field that can rescue the women affected by cancerous tissues. A modest and skilful approach in microwave imaging (MWI) for data inversion is proposed. MWI in medicinal applications has been famous in last two decades and presently it is using for the detection of breast cancer. Medical imaging by microwave technology has been potential to investigate the electrical distribution, dielectric properties, and as well conductivity for malignant tumor inside the breast. In this study subscription, the writers have reviewed the research importance for inverse scattering method in the microwave tomographic imaging as well as discussed Born type approximation for malignant tumor detection. Distorted Born iterative method (DBIM) will also be discussed. This paper presents the approaches of image reconstruction for breast cancer detection.
\end{abstract}

Keywords: Microwave Tomography, Breast cancer Detection, Distorted Born iterative method (DBIM)

\section{Introduction}

Microwave imaging (MWI) is going to one of the main pillars in biomedical fields of comprehensive cancer care. The advantages of MWI are non invasive, diagnosis without any destruction of tissues, unharmed microwaves and can be overcome of the large amount of time-consuming for the biomedical treatment. The purpose of MWI is, to collect the information from the interrogative microwaves at specific frequencies. This method is supposed to be an emerging method for a long time. In several fields, like biomedical engineering, geophysical prospecting and civil and industrial engineering, MWI has been proven to provide the information for diagnose the problem. It is important that to motivate for the improvement in microwave technology for the detection of breast tumors [1].

In order to treat the malignant tumor is needed to be detecting in its early stage [24]. The restrictions of traditional magnetic resonance imaging (MRI) and X-ray mammography are well-known and in reaction to these restrictions, a lot of perfect modes for the detection of malignant tumor are under development $[24,25]$. However, presently X-ray's technique is very famous and considering as a gold standard technique for this purpose. On the other side, MRI and ultrasound techniques are very expensive and less effective in case of mass screening. For the further details of X-ray mammography readers refer to [2]. However, Xrays mammography and other techniques are not able to detect the tumor in its early stage. It is noticed that ionized radiations are accumulated due to several scans in X-ray's technique.

MWI methods in case of early breast cancer detection give charming and alternative techniques over conventional $\mathrm{x}$-ray mammography. The motivation to this method is due to the investigation of conductivity and dielectric properties between healthy and cancerous tissues at different frequencies (healthy is $2: 1$ and malignant tumor is 10:1) [3]. The best 
way of this method is very comfortable and painless as compared to other methods like $\mathrm{x}$ ray etc. and there is non ionizing radiation in microwave imaging methods. For detailed information see [4].

Although super-resolution has been observed and attributed to evanescent waves in near-field measurements or in multiple-scattering environments $[5,6]$, microwave detection of early-stage malignancies is nevertheless challenged by the moderate endogenous dielectric contrast, the small scattering area of these malignancies, and the heterogeneous scattering environment of healthy glandular tissue in which tumors often form.

The three-dimensional (3D) imaging results presented in $[7,8]$ were obtained with a frequency-domain inverse scattering algorithm formulated using the distorted Born iterative method (DBIM) [26]. The imaging method was evaluated in [8], in application to realistic numerical breast phantoms of healthy tissues over a range of fibroglandular density classifications. The method was shown to produce representative reconstructions of the actual profile of complex permittivity, suggesting that it may be suitable for normal tissue density assessment. However, the low resolution of the images suggests that earlystage malignancies may not be identifiable by direct inspection of the images. Another class of numerical technique is then considered, which is stated in radar terminology as inverse scattering methods. The object profile is reconstructed by solving the inverse scattering problem using iterative methods. In addition to obtaining the shape as the object, a quantitative sketch of the dielectric constant profile is also obtainable, which is extremely valuable for diagnostic information.

This paper is drafted in a following sequential manner. Section II presents the review on inverse scattering in microwave imaging for breast cancer detection. Section III and IV present concept and implementation of Born iterative method of inverse scattering and non-linear inverse scattering method are presented in section $\mathrm{V}$, and paper concludes with some recommendations and references.

\section{Review on Inverse Scattering in Microwave Imaging for Breast Cancer}

Scattering in microwave imaging are discussed throughout this section.

A $2.45 \mathrm{GHz}$ microwave camera was developed by Franchois [9] where they used method of moments combined with the distorted Born iterative methods for image reconstruction. In microwave imaging, the algorithm developed by Sourov [10], the direct solution for the scattering problem was obtained by fast forward iterative method and inverse solution by Newton iterative method. Chew [9] developed a time-domain approach that incorporated the FDTD method in distorted Born iterative method to the reconstruct images. An iterative algorithm to solve the non-linear inverse scattering problem for twodimensional microwave tomographic imaging using time domain scattering data was developed by Moghaddam [10]. The method was based on performing born iterations on volume integral equation and then successively calculating higher-order approximations to the unknown object profile. Wide band time -domain scattered field measurements made it possible to use sparse data sets, and thus reduced experimental complexity and computation time. Tijhuis [29] conducted 2-D inverse profiling with the Ipswich data provided by the Institute of Fresnel, France. The distorted born iterative approach was applied to the image reconstruction. They observed that the dynamic range was large and resolution was limited for low frequency, whereas at high frequencies, the resolution was increased as the cost of dynamic range. They also developed a technique for solving 2-D inverse scattering problem in the microwave tomographic imaging by parameterize the scattering configuration and determining the optimum value for the parameters by minimizing a cost function involving the known scattered fields [11]. The efficiency of the computation was improved by using CGFFT iterative scheme. 
Born iterative method was suggested for solving the inverse scattering problem in the microwave tomographic imaging by Wang [27]. The Green's function was kept unchanged in the iterative procedures. As the method failed for imaging strong scatterers, they proposed an alternative method named distorted Born iterative method [12] wherein the Green's function, and the background mediums were updated in every iteration. The new method showed faster convergence rate. Liu [10] developed fast forward and inverse methods to simulate 2-D microwave imaging for breast cancer detection. The forward methods were based on extended Born approximation, fast Fourier transforms, conjugate gradient and bi-conjugate gradient methods. The inverse methods were based on a two-step non-linear inversion. Numerical results demonstrated the skill of the algorithm. The work was extended [14] to 3-D imaging of the breast using stabilized biconjugate gradient technique (BiCGSTAB) and FFT algorithm, to compute electromagnetic fields. The computational domain of the electric field integral equation was discretized by simple basis functions through the weak form discretization.

Multiple frequency information was utilized for performing microwave image reconstruction of tissue property, dispersion characteristics by Fang [15]. They adopted Gauss-Newton iterative strategy, which facilitated the simultaneous use of multiple frequency measurement data in a single image reconstruction. Caorsi [16] proposed a multi-illumination multi view approximation for 2-D microwave imaging based on Genetic algorithm. The inverse problem was recast as an optimization problem, solved in the frame work of Born approximation.

Multiplicative regularization scheme was introduced to deal about the problem of detection and imaging of homogeneous dielectric objects by Abubakar [17]. Contrast source inversion method was introduced for reconstructing the complex index of refraction of a bounded object immersed in a known background medium by Van den Berg [18]. The method accommodated spatial variation of the incident fields and permitted a-priori information about the scatterer. Newton- Kantorovich iterative reconstruction algorithm was developed for microwave tomography by Joachimowicz [19]. The results of biological imaging provided a quantitative estimation of the effect of experimental factors such as temperature as the immersion medium, frequency and signal to noise ratio.

Multi frequency scattering data was utilized for Image reconstruction in 2-D microwave imaging by Belkebir[20]. Newton-Kantorovich (NK) and Modified Gradient (MG) methods were used for image reconstruction. Both methods used a-priori information, and the same initial guess that the characteristic function was non-negative. Semenov [28, 21] developed a 2-D quasi real time tomographic system for microwave imaging. The Rytov approximations were modified to take into account of the radiation method as well as the locations of the antenna for 2-D microwave tomography. Images of water immersed gel phantom were developed. Better results were reported with objects of high contrast. The same group also [22] developed vector Born reconstruction method for improving 3-D microwave tomographic images. The method utilized only one component to the vector electromagnetic filed for image reconstruction. They also studied Newton's method for reconstructing 2-D images of breast phantoms and Modified Gradient method for reconstructing 3-D images [23].

\section{Inverse Scattering in Microwave Imaging}

In inverse scattering, scattered data from the target collected from measurement domain and then with the help of this data construct the desired image. Additionally, for acquiring the size and shape of the tumor, a detailed description of the dielectric properties and conductivity can be achieved by inverse scattering method. Furthermore, it (inverse scattering) depends on the number of scattering take place inside the target. The non linearity occurs due to these numbers of scattering into the scattered field which is related to the object function, and this object function gives information about the dielectric 
properties and conductivity distributions of the malignant tumor. However, numerically we can solve these equations by using different methods such as Born iterative method, Rytov approximation, etc. The configuration inverse scattering method is given in Figure 1.

As it is well known, the relation between target and scattered field is non linear. So, the non linearity occurs due to number of scattering after striking the target is shown in Figure 2.

When only scatterer ' 1 ' is present, the scattered field is $\bar{E} 1$ s and when only scatterer ' 2 ' is present the scattered field is $\overline{\mathrm{E}} 2 \mathrm{~s}$. However when both the scatterers are simultaneously present, the scattered field is $\bar{E} 1 s+\bar{E} 2 s+\bar{E} m s$ where $\bar{E} m s$ is a result of multiple scattering between the scatterers.

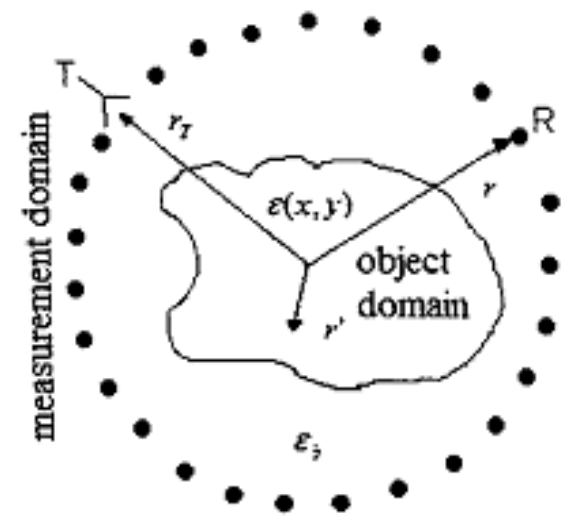

Figure 1. The Configuration Inverse Scattering Method

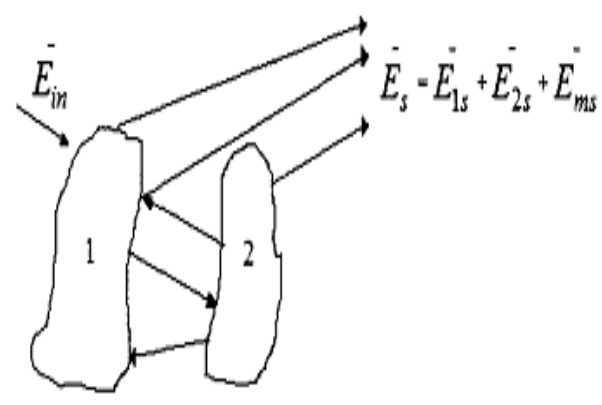

Figure 2. Scattering Pattern

\subsection{Non-linear Inverse Scattering Method}

Non-linear inverse scattering theories deal with problems involving multiple scattering effects. [25]

The scattering by an inhomogeneous body is represented by the equation is given by,

$$
E_{\text {scat }}(\bar{r})=\omega^{2} \mu \int_{v} g\left(\bar{r}, \bar{r}^{\prime}\right) \delta \varepsilon\left(\bar{r}^{\prime}\right) E\left(\bar{r}^{\prime}\right) d S
$$

As we know that

$$
E_{t}(\bar{r})=E_{\text {scat }}(\bar{r})+E_{\text {inc }}(\bar{r})
$$

$\overline{\mathrm{r}}$ stands for a point in the measurement domain and $\overrightarrow{\mathrm{r}}$ for the object domain. In general it is impossible to solve the non linear equation; hence some approximations need to be made. The Born Approximation is the simpler approach to linearize this inverse scattering equation.

Eqn. 1 shows that total field $\mathrm{E}(\overrightarrow{\mathrm{r}})$ is expressed as the sum of the incident field Einc $(\overrightarrow{\mathrm{r}})$ and a small perturbation Escat $(\overrightarrow{\mathrm{r}})$. In a similar way we can write as,

$$
E_{\text {scat }}(\bar{r})=E_{t}(\bar{r})+\omega^{2} \mu \int_{v} g\left(\bar{r}, \bar{r}^{\prime}\right) \delta \varepsilon\left(\bar{r}^{\prime}\right) E\left(\bar{r}^{\prime}\right) d S
$$

If the scattered field Escat $(\overrightarrow{\mathrm{r}})$ is small compared to the incident field Einc $(\overrightarrow{\mathrm{r}})$,the effects of the second integral can be ignored to arrive at the approximation,

$$
\mathrm{E}_{\mathrm{scat}, \mathrm{b}}(\overline{\mathrm{r}})=\omega^{2} \mu \int_{v} g_{b}\left(\bar{r}, \bar{r}^{\prime}\right) \delta \varepsilon\left(\bar{r}^{\prime}\right) E_{i n c, b}\left(\bar{r}^{\prime}\right) d S
$$


This constitutes the first order Born Approximation. This approximation is valid only if the magnitude of the scattered field is smaller than the incident field. Hence the incident field can be considered to that generated by a uniform line source, which is given by the Hankel function as,

$$
E_{\text {inc }}\left(\overline{r_{T}} / \bar{r}^{\prime}\right)=\frac{j}{4} H_{o}^{1}\left(k_{o}\left(\overline{r_{T}} / \bar{r}^{\prime}\right)\right)
$$

For eqn. 3, it is assumed that the medium is homogeneous and hence Green's function is known in the closed form. The field inside the scatterer is found numerically for an initial guess of the permittivity profile. Then an improved profile is obtained by comparing the differences between the estimated and the measured scattered field. This process is repeated until a convergent solution is reached. This iteration procedure is called as the Born iterative method. While the Born iterative method is simple to implement, it does not offer second order convergence and hence not suitable to solve non linear inverse scattering problem.

Hence Distorted Born Iterative Method is developed for the image reconstruction of breast tissues. The method is described in detail in the following section. Here we only discuss distorted Born iterative method (DBIM).

\subsection{Distorted Born Iterative Method (DBIM)}

The very important quantitative approach is the distorted Born iterative method (DBIM) in reconstruction of image. In this method, the back ground medium is considered inhomogeneous and is updated with each iteration. Hence the equation for Green's function and the equation for incident field are updated with each iteration. The wave equation for the inhomogeneous medium can be written as,

$$
\left(\left(\nabla^{2}+k_{b}^{2} E(\bar{r})\right)=0\right.
$$

Where the wave number kb represents the spatial frequency of the plane wave in the medium and is a function of the wavelength $\lambda$ or $\mathrm{kb}=2 \Pi / \lambda=\omega \sqrt{\mu \varepsilon b}$. A solution to this equation is given by a plane wave as,

$$
E(\bar{r})=e j \bar{k} b \bar{r}
$$

Which is on simplification using eqn. 1 and using the Green's function becomes,

$$
E(\bar{r})=E_{\text {inc, } b}(\bar{r})+\omega^{2} \mu \int_{v} g_{b}\left(\bar{r}, \bar{r}^{\prime}\right) \delta \varepsilon\left(\bar{r}^{\prime}\right) E\left(\bar{r}^{\prime}\right) d S
$$

where $\bar{r}$ stands for a point in the measurement domain and $\bar{r}$ for the object domain. $\varnothing(\vec{r})$ is the total field at $\bar{r}$, Øinc, $b(\vec{r})$ is the incident field measured at $\bar{r}$ in the presence of the background inhomogeneity, $\omega$ represents the angular frequency and $\mu$ the permeability. As biological materials are non $>$ magnetic, the relative permeability is unity and hence $\mu=\mu \mathrm{o}$ the permeability of free space.

The $\delta \varepsilon(\vec{r})$ is defined as

$$
\delta \varepsilon(\overrightarrow{\mathrm{r}}) \varepsilon(\overline{\mathrm{r}})-\varepsilon_{\mathrm{b}}(\overrightarrow{\mathrm{r}})
$$

where $\varepsilon(\overrightarrow{\mathrm{r}})$ is the complex relative permittivity of the object and $\varepsilon(\overrightarrow{\mathrm{r}})$ is the complex relative permittivity of the background medium. $g b(\overrightarrow{\mathrm{r}}, \overrightarrow{\mathrm{r}})$ is the Green's function and $\emptyset(\overrightarrow{\mathrm{r}})$ is the total field inside the scatterer. The integral term in Eqn. 7 is the scattered field due to the dielectric contrast of the scatterer. Hence, 


$$
\emptyset_{\text {sac,b}}(\overrightarrow{\mathrm{r}})=\varnothing(\overrightarrow{\mathrm{r}})-\emptyset_{\text {inc,b}}(\overrightarrow{\mathrm{r}})
$$

Therefore

$$
E_{\text {scat }, b}(\bar{r})=\omega^{2} \mu \int_{v} g_{b}\left(\bar{r}, \bar{r}^{\prime}\right) \delta \varepsilon\left(\bar{r}^{\prime}\right) E\left(\bar{r}^{\prime}\right) d S
$$

This scattered field is a non-linear functional of $\delta \varepsilon\left(\overline{\mathrm{r}}^{\prime}\right)$ because $\emptyset\left(\overline{\mathrm{r}}^{\prime}\right)$ in the integral is also a function of $\delta \varepsilon\left(\overline{\mathrm{r}}^{\prime}\right)$. In the inverse scattering problem, $\delta \varepsilon\left(\overline{\mathrm{r}}^{\prime}\right)$ need to be solved from the measurement of the scattered field outside the scatterer. To linearize the non-linear relation between the scattered field and the object function, it is assumed that $\varepsilon(\overrightarrow{\mathrm{r}})$ is close to $\varepsilon b(\vec{r})$. Hence $Ø s a c, b(\vec{r})<<\varnothing$ inc, $b(\vec{r})$. Therefore $\varnothing(\vec{r})$ in Eqn. 10 is approximated with Øinc,b( $\overrightarrow{\mathrm{r}})$, which is the incident field inside the scatterer. This approximation is called as distorted Born approximation. The scattered field is written as

$$
E_{\text {scat }, b}(\bar{r})=\omega^{2} \mu \int_{v} g_{b}\left(\bar{r}, \bar{r}^{\prime}\right) \delta \varepsilon\left(\bar{r}^{\prime}\right) E_{i n c, b}\left(\bar{r}^{\prime}\right) d S(11)
$$

Substituting Eqn. 7 in Eqn. 11,

$$
\mathrm{E}_{\text {scat }, \mathrm{b}}(\overline{\mathrm{r}})=\omega^{2} \mu \int_{v} g_{b}\left(\bar{r}, \bar{r}^{\prime}\right)\left(\varepsilon_{r}\left(\bar{r}^{\prime}\right)-\varepsilon_{r b}\left(\bar{r}^{\prime}\right) E_{\text {inc,b }}\left(\bar{r}^{\prime}\right) d S\right.
$$

Where $\mathrm{k}_{\mathrm{o}}^{2}=\omega^{2} \mu \varepsilon_{\mathrm{o}}$. The singularity that exists in the Green's function when observation point is in the object domain, is taken care by using Richmond approximation where the square cells in the computation domain are approximated with circular cells of same cross sectional area. Hence $k_{o}^{2}=\int_{v} d S g_{b}\left(\bar{r}, \bar{r}^{\prime}\right)$ in Eqn. 12 is modified as,

$$
\begin{array}{rr}
\frac{j \pi k_{0}}{2} \sum_{n=1}^{N} a_{n} J_{1}\left(\mathrm{k}_{\mathrm{b}} \mathrm{a}_{\mathrm{n}}\right) \mathrm{H}_{\mathrm{o}}^{(1)}\left(\mathrm{k}_{\mathrm{b}}\left(\overline{\mathrm{r}}-\overline{\mathrm{r}}^{\prime}\right)\right) & \text { for } \overline{\mathrm{r}} \neq \overline{\mathrm{r}}^{\prime} \\
\frac{j \pi k_{0}}{2} \sum_{n=1}^{N} a_{n} \mathrm{H}_{\mathrm{o}}^{(1)}\left(\mathrm{k}_{\mathrm{b}} \mathrm{a}_{\mathrm{n}}\right)-2 \mathrm{~J} & \text { for } \overline{\mathrm{r}} \neq \overline{\mathrm{r}}^{\prime}
\end{array}
$$

Where $\mathrm{N}$ stands for the total number of cells in the computation domain and $\mathrm{a}_{\mathrm{n}}$ the radius of the circular cell. $\mathrm{H}_{\mathrm{o}}^{(1)}$ represents zero order of Hankel function of first kind, $\mathrm{H}_{1}^{(1)}$ represents first order Hankel function of first kind and $\mathrm{kb}$ represents the wave number $\omega=\sqrt{\mu \varepsilon_{\mathrm{b}}} \omega$.

In 2-D, the incident field inside the scatterer is written using eqn. 5.19 as,

$$
E_{\text {inc }, b}\left(\bar{r}^{\prime}\right)=\frac{j}{4} \mathrm{H}_{\mathrm{o}}^{(1)} \mathrm{k}_{\mathrm{b}}\left(\overline{\mathrm{r}}-\overline{\mathrm{r}}_{\mathrm{T}}{ }^{\prime}\right)
$$

where $\overline{\mathrm{r}}_{\mathrm{T}}$ represents the transmitter location. Eqns. 13 - 15 are substituted in the linearized eqn. 12 and is solved numerically to obtain the value of $\delta \varepsilon(\overrightarrow{\mathrm{r}})$. This method to obtain $\delta \varepsilon(\overrightarrow{\mathrm{r}})$ from Øsac,b( $\overrightarrow{\mathrm{r}})$ is termed as the inverse scattering solution . $\varepsilon \mathrm{b}(\overrightarrow{\mathrm{r}})$ can be calculated from $\delta \varepsilon(\overrightarrow{\mathrm{r}})$ using Eqn. 8. This value of $\varepsilon(\overrightarrow{\mathrm{r}})$ is then used as the new . $\varepsilon b(\overrightarrow{\mathrm{r}})$ and using this, the incident field and Green's function inside the object are updated. The total field inside the scatterer is then updated using the equation,

$$
E(\bar{r})=E_{\text {inc }, b}(\bar{r})+\omega^{2} \mu \int_{v} g_{b}\left(\bar{r}, \bar{r}^{\prime}\right) \delta \varepsilon\left(\bar{r}^{\prime}\right) E\left(\bar{r}^{\prime}\right) d S
$$


Computing $\varnothing(\overrightarrow{\mathrm{r}})$ using Eqn. 16 is termed as the forward scattering solution. This value of $\varnothing(\vec{r})$ is then substituted as Øinc,b( $(\vec{r})$ in the Eqn. 11.

The entire procedure is repeated by first obtaining the inverse scattering solution and then the forward scattering solution. This iterative procedure where the dielectric properties of the background medium are also updated with each iteration is called as the distorted Born iterative method. 


\section{Discussion}

In contrast, in an inverse scattering problem, the goal is to determine the physical quantities of the media $\left(\varepsilon_{\mathrm{r}}, \sigma\right)$ from the knowledge of the electric field $(\overline{\mathrm{E}})$ at a set of receiver points and knowledge of the source $(\bar{J})$.

Finally, microwave tomographic imaging is showing a significant promise as a new method for the early detection of breast cancer. It contains the measurements of the scattered microwave signals through the contrast media and simulates these signals numerically using a breast model. The corresponding inverse problem includes in minimizing the mismatch between the signal of the scattered waves and the one simulated with the numerical model, undertaking at the same time the corresponding corrections of the initial modeling inverse scattering we measure incident electric field and scattered electric field. Review shows that extensive research goes on in the field of microwave tomographic imaging to solve the inverse scattering problem, to realize a viable system for medical imaging. Most of the works reported were simulations, and a few were tested tentatively on phantoms. Reconstruction of 2-D tomographic images from experimentally collected scattered fields on breast tissues (normal tissue with cancerous inclusion) in the presence of a matching coupling medium is not tried somewhere else.

The present chapter analyses distorted Born iterative method to solve the inverse scattering problem using experimentally collected scattered data on breast tissues and on breast phantoms. This method is adopted as it is simple, easy to implement and could develop images with reasonable accuracy, even for strong scatterers.

\section{Conclusion}

In this paper author present a suitable, inexpensive and exact method for the detection of malignant tumor inside the breast by MWI. This article showed the research status and result of bowtie antenna at $3 \mathrm{GHz}$ frequency which will use in our future work. This antenna can be use in microwave tomography technique because it's working and efficiency in this field is perfect. Finally, it is important to remember that in addition to microwave imaging, several other alternative breast cancer detection modalities are actively being pursued, including optical imaging methods. However for future work, with the help of this antenna need to construct a bra-shaped device for this purpose.

\section{Acknowledgment}

The Authors are thankful to china Scholarship council and Northwestern Polytechnical University Xi' an, China for the research support.

\section{References}

[1] J. A. Harvey and V. E. Bovbjerg, "Quantitative assessment of mammographic breast density: Relationship with breast cancer risk, Radiology, vol. 230, (2004), pp. 29-41.

[2] E. C. Fear, X. Li, S. C. Hagness and M. Stuchly, "Confocal microwave imaging for breast cancer detection: localizations of tumors in three dimensions", IEEE Transactions on Biomedical Engineering, vol. 49 , no. 8 , pp. 812-822.

[3] S. C. Hagness, A. Taflove and J. E. Bridges, "Two-dimensional FDTD analysis of a pulsed microwave confocal system for breast cancer detection: fixed-focus and antenna-array sensors", IEEE Transactions on Biomedical Engineering, vol. 45, no. 12, pp. 1470-1479.

[4] E. Fear, P. Meaneya and M. Stuchly, "Microwaves for breast cancer detection?", IEEE Potentials, vol. 22, no. 1, (2003), pp. 12-18.

[5] L. V. Wang, X. Zho, H. Sun and G. Ku, "Microwave-induced acoustic imaging of biological tissues", Rev. Sci. Instrum., vol. 70, (1999), pp. 3744-3748.

[6] F. Chen and W. C. Chew, "Experimental verication of super resolution in nonlinear inverse scattering", Appl.Phys. Lett., vol. 72, (1998), 3080-2. 
[7] X. Li, E. J. Bond, B. D. Van Veen and S. C. Hagness, "An overview of ultrawideband microwave imaging via space-time beamforming for early-stage breast cancer detection", IEEE Antennas Propag. Mag., vol. 47, (2005), pp. 19-34.

[8] J. M. Sill and E. C. Fear, "Tissue sensing adaptive radar for breast cancer detectionexperimental investigation of simple tumor models", IEEE Trans. Microw, Theory Tech., vol. 53, (2005), pp. 3312-9.

[9] A Franchois, A. Joisel, C. Pichot and J. C. Bolomey, "Quantitative microwave imaging with a $2.45 \mathrm{GHz}$ planar microwave camera", IEEE Transactions on Medical Imaging, vol. 18, (1998), pp. 550-561.

[10] A. E. Souvorov, A. E..Bulyshev, S. Y. Semenov, R. H. Svenson, A. G. Nazarov, Y. E. Sizov and G. P. Tatsis, "Microwave Tomography: A two-dimensional Newton iterative scheme", IEEE Transactions on Microwave Theory and Techniques, vol. 46, (1998), pp. 1654-1658.

[11] M. Moghaddam and W. C. Chew, "Non linear two dimensional velocity profile inversion using time domain data", IEEE Transactions on Geoscience and Remote Sensing, vol. 30, (1992), pp. 147-156.

[12] A. G. Tijhuis, K. Belkebir, A. S. Litman and B. P. de Ron, "Theoretical and computational aspects of 2D inverse profiling”, IEEE Transactions on Geoscience and Remote Sensing, vol. 39, (2001), pp. 13161329.

[13] Y. M. Wang and W. C. Chew, "An iterative solution of the twodimensional electromagnetic inverse scattering problem", International Journal of Imaging Systems and Technology, vol. 1, (1989), pp. 100108.

[14] W. C. Chew and Y. M. Chang, "Reconstruction of two-dimensional permittivity distribution using the distorted Born iterative method", IEEE Transactions on medical Imaging, vol. 9, (1991), pp. 218-225.

[15] Q. R. Liu, Z. Q. Zhang, T. T. Wang, J. A. Bryan, G. A. Ybarra, L. W. Nolte and A. T. Joines, "Active microwave imaging I - 2 D forward and inverse scattering methods", IEEE Transcations on Microwave Theory and Techniques, vol. 50, (2002), pp. 123-132.

[16] Q. R. Liu, Z. Q. Zhang, T. T. Wang, C. Xiao, E. Ward, G. Ybarra and A. T. Joines, "Active microwave imaging 11- 2 D reconstruction and a 3-D fast volume integral equation method", IEEE Transactions on Microwave Theory and Techniques, vol. 50, (2002), pp. 223-232.

[17] Q. Fang, P. M. Meaney and K. D. Paulsen, "Microwave image reconstruction of tissue property dispersion characteristics utilizing multiple frequency information", IEEE Transactions on Microwave Theory and Techniques, vol. 52, (2004), pp. 1866-1875.

[18] S. Caorsi and M. Pastorino, "Two-dimensional microwave imaging approach based on a genetic algorithm”, IEEE Transactions on Antennas and Propagation, vol. 48, (2000), pp. 370-373.

[19] A. Abubakar and P. M. Van den Berg, "The contrast source inversion method for location and shape reconstruction", Inverse Problems, vol. 18, (2002), pp. 495-510.

[20] P. M. Van den Berg and A. Abubakar, "A contrast source inversion method", Inverse Problems, vol. 13, (1997), pp. 1607-1620.

[21] K. Belkebir, R. E. Kleinman and C. Pichot, "Microwave imaging-location and shape reconstruction from multifrequency scattering data", IEEE Transactions on Microwave Theory and Techniques, vol. 45, (1997), pp. 469-476.

[22] S. Y. Semenov, R. H. Svenson, A. E. Boulyshev, A. E. Souvorov, A. G. Nazarov, Y. E. Sizov, V. Y. Borisov, V. G. Posukh, I. M. Kozlov, A. G. Nazarov and G. P. Tatsis, "Microwave Tomography: experimental investigation of the iteration reconstruction algorithm", IEEE Transactions on Microwave Theory and Techniques, vol. 46, (1998), pp. 133-141.

[23] S. Y. Semenov, R. H. Svenson, A. E. Boulyshev, A. E. Souvorov, V. Y. Borisov, A. N. Starostin, K. R. Dezen, G. P. Tatsis and V. Y. Baranov, "Microwave Tomography: two dimensional system for biological imaging", IEEE Transcations on Microwave Theory and Techniques, vol. 43, (1996), pp. 869877.

[24] Mammography and Beyond: Developing Techniques for the Early Detection of Breast Cancer. Washington, DC: National Academy, (2000).

[25] P. T. Huynh, A. M. Jarolimek and S. Daye, "The false-negative mammogram, Radiography", vol. 18, no. 5, (1998), pp. 1137-1154.

[26] C. K. Kuhl, MRI of breast tumors Eur. Radiol. 10 46-58.

[27] A. G. Tijhuis, K. Belkebir, A. S. Litman and B. P. de Ron, "Multuiple frequency distorted Born approach to 2-D inverse profiling", Inverse Problems, vol. 17, (2001), pp. 1635-1644.

[28] N. Joachimowicz, J. J. Mallorqui, J. C. Bolomey and A. Broquetas, "Convergence and stability assessment of Newton-Kantorovich reconstruction algorithms for microwave tomography", vol. 17, (1998), pp. 562-569.

[29] W. C. Chew, "Imaging and inverse problems in electromagnetics", Advances in Computational Electrodynamics: The Finite Difference Time Domain Method, ATaflove, Ed. Norwood, MA: Artech House, Chapter 12, (1998). 
International Journal of Signal Processing, Image Processing and Pattern Recognition Vol.7, No.1 (2014) 